\title{
SEA LICE VACCINE DEVELOPMENT BASED ON AN IMMUNOGENIC PEPTIDE DERIVED FROM THE RIBOSOMAL PROTEIN PO
}

\author{
Yamila Carpio', Janet Velazquez ${ }^{1}$, Yeny Leal ${ }^{1}$, Naylin Herrera ${ }^{1}$, Claudia García ${ }^{1}$, \\ Jannel Acosta ${ }^{1}$, Antonio Morales ${ }^{1}$, Fumio Takizawa ${ }^{2}$, Oriol Sunyer ${ }^{2}$, Mark Fast ${ }^{3}$, \\ Mario Pablo Estrada ${ }^{1^{* *}}$ \\ ${ }^{1}$ Centro de Ingeniería Genética y Biotecnología (CIGB), Habana, Cuba \\ ${ }^{2}$ School of Veterinary Medicine, University of Pennsylvania, USA \\ ${ }^{3}$ Atlantic Veterinary College, University of Prince Edward Island, Canada
}

\begin{abstract}
Sea lice (Copepoda, Caligidae) are the most widely distributed marine pathogens in the salmon industry. Vaccination is considered an efficient, environmentally safe and economically sustainable alternative for control of ectoparasite infestations. Although emerging sea lice proteins have been identified recently, and have been proposed as potential targets for generating protective molecules, only a limited number of them have been evaluated in vaccine trials with unsuccessful results. On the other hand, more than 80 proteins are found in eukaryotic ribosomes. The protein $\mathrm{P} 0$ is essential for the assembly of the 60S ribosomal subunit and essential for cell viability. A vaccinationchallenge trial with an immunogenic peptide of Rhipicephalus sanguineus protein P0 reduced survival of ticks with an overall efficacy of $90 \%$, suggesting that it might be a promising antigen candidate for the control of ectoparasite. We have identified an immunogenic region of the ribosomal protein P0 from Caligus rogercresseyi and Lepeophtheirus salmonis that is not very conserved compared to host $\mathrm{P} 0$. We developed several vaccine candidates based on this peptide and produced in E. coli. These antigens were able to elicit a high specific antibody response after intraperitoneal (ip) immunization using tilapia as teleost fish model. It has been shown that ip immunization with some antigens cannot significantly reduce larval lice numbers despite enhanced specific antibody titers were raised. In this context, we have been developed for first time several tools to monitor tilapia immune response (IgT, IFN- $\gamma$, IL-4, CD154) to vaccination in order to perform a more complete evaluation of the impact of the vaccinate candidates on the fish immune system. These findings will be finally validated in an immunization-challenge trial in Salmo salar. The overall results are relevant in the development of an effective vaccine against sea lice.
\end{abstract}

\section{KEYWORDS}

Sea lice, vaccine, immune response, cytokines, ribosomal protein

**Corresponding author. Phone: (53-7)2504423; Email address: mario.pablo@cigb.edu.cu 